\title{
Improving Medication Adherence: Keep Your Eyes on the Prize
}

\author{
Zachary A. Marcum, PharmD, $P h D^{7}$ and Walid F. Gellad, MD, MPH $H^{2,3}$ \\ ${ }^{1}$ School of Pharmacy, University of Washington, Seattle, WA, USA; ${ }^{2}$ Division of General Internal Medicine and Center for Pharmaceutical Policy and \\ Prescribing, University of Pittsburgh, Pittsburgh, PA, USA; ${ }^{3}$ VA Pittsburgh Healthcare System, Pittsburgh, PA, USA.
}

$\mathrm{J}$ Gen Intern Med 32(3):236-7

DOI: $10.1007 / \mathrm{s} 11606-016-3927-4$

(C) Society of General Internal Medicine 2016

$\mathrm{M}$ edication adherence is a set of complex health behaviors that challenges patients of all ages and health conditions. Researchers have spent decades trying to improve medication adherence, and yet these interventions have been wrought with limitations and sobering findings. The most recent Cochrane systematic review of the impact of adherence interventions found that effects were inconsistent across studies and that only a small number of interventions led to a modest improvement in both adherence and clinical outcomes. ${ }^{1}$ The authors concluded that existing adherence interventions "are mostly complex and not very effective." 1 In addition, the authors recommended that future research include robust study designs of feasible long-term interventions with sufficient study power to detect improvements in patientcentered clinical outcomes. $^{1}$

A key issue in implementing any adherence intervention is ensuring valid measurement of adherence. Measurement of medication adherence is complex, since most measures serve as proxies for medication-taking behavior. ${ }^{2}$ For example, pharmacy claims data are increasingly used for conducting adherence research, although the pharmacy claim serves only as a proxy for medication consumption, and in reality it is simply measuring refills. Other methods for measurement, including self-report, pill counts, and electronic capture of pill bottle opening, also require important assumptions about the relationship between the measure and actual medication-taking. It is further assumed that improving medication adherence will improve process measures such as blood pressure or cholesterol control, which will in turn improve the health outcome(s) of patients including quality of life and mortality.

In this issue of JGIM, Reddy and colleagues present findings on the effect of daily pill bottle alarms combined with individual or partner feedback reports on statin medication adherence. $^{3}$ This 3-month, three-arm randomized clinical trial randomized 126 veterans with known coronary artery disease and poor adherence (medication possession ratio [MPR] $<80 \%$ ) to one of three groups: (1) a control group that received a pill-monitoring device (i.e., a GlowCap ${ }^{\circledR}$ bottle) with no alarms or feedback; (2) an individual feedback group

Published online November 15, 2016 that received a daily alarm and a weekly medication adherence feedback report; and (3) a partner feedback group that received an alarm and a weekly feedback report that was shared with a friend, family member, or a peer. By the end of the 3-month intervention, statin adherence was significantly higher in both the individual and partner feedback groups compared to the control arm (89\% and $86 \%$ vs. $67 \%$, respectively). However, at 6 months, statin adherence did not differ among the groups (60\% and $52 \%$ vs. $54 \%$ ).

The study had a number of strengths and unique aspects. First, the use of a feedback component in the intervention arms was a novel approach. The authors were thus able to assess the impact of peer support (via social forces) or individual feedback on medication adherence behavior above and beyond the reminder effects of the GlowCap ${ }^{\circledR}$ bottle. The use of peer support is increasing in behavior change interventions ${ }^{4}$, and the application to adherence interventions is important and should be further investigated. Second, the study included only patients who had suboptimal medication adherence at baseline as measured by a 16-month statin MPR <80\%. In other words, the study targeted patients who had a documented need for intervention on some level. Interventions targeting individuals with medication adherence problems can have modest but significant effects on medication-taking behavior. ${ }^{5}$ Additional research is needed to further test this and other riskstratification approaches for improving medication adherence and the targeting of interventions.

Despite these strengths, some limitations deserve mention, most notably concerns about adherence measurement. The medication adherence measure was the GlowCap ${ }^{\circledR}$ bottle, which includes a computer chip in the lid that detects and time-stamps when the lid is removed. This is similar to the Medication Event Monitoring System (MEMS), which is considered a "gold standard" measure of medication adherence in trial settings. ${ }^{6}$ However, it is important to remember that it is an indirect method of measurement-opening the bottle does not equal taking the statin. It is entirely plausible, as the authors discuss, that patients receiving feedback reports were more likely to open the bottle but no more likely to take the medication.

These concerns are magnified given the lack of change in LDL seen in the intervention groups. Despite the positive impact of the intervention on opening the pill bottle, it was found to have no significant effect on LDL levels at 6 months. In fact, the control group had numerically greater absolute 
LDL reduction $(9.1 \mathrm{mg} / \mathrm{dl})$ than those in the individual feedback group $(5.0 \mathrm{mg} / \mathrm{dl})$ and the partner feedback group $(4.6 \mathrm{mg} / \mathrm{dl})$. It is not clear why this finding occurred. It is possible that certain unmeasured behaviors, such as diet and/or exercise, were different between the control group and interventions arms. In addition, the null finding on LDL reduction at 6 months may reflect a lack of difference in adherence among the three groups at that time point, rather than during the intervention period. Without more frequent measures of LDL during the trial, it is difficult to interpret the intervention's impact on this process measure.

There is emerging consensus that multiple methods of adherence measurement should be included in studies of adherence interventions, with comparison of results across methods. ${ }^{7}$ A review of studies comparing the MEMS with other methods for measuring medication adherence reported that, compared to MEMS, adherence was overestimated by $17 \%$ using self-report, by $8 \%$ using pill count, and by $6 \%$ using rating by a surrogate. ${ }^{6}$ Although self-reported medication adherence may overestimate the degree of adherence, it can offer critical insight into patient-specific barriers to medication-taking. ${ }^{8}$ For example, the intervention studied by Reddy et al. would likely have a greater impact on patients whose main barrier is forgetting to take their medication than on patients whose main concern is cost-related. Thus, selfreported adherence data can allow for the delivery of patienttailored interventions. Reddy and colleagues measured selfreported statin adherence at baseline using the four-item Morisky Medication Adherence Scale. This information was used as an adjustment variable, whereas it could also potentially have been used as an inclusion criterion.

Despite these limitations, the study shines an important light on the continued need for sustainable medication adherence interventions. The authors cite previous research suggesting that formation of a habit takes 66 days on average; thus their selection of a 3-month intervention more than covered this time frame. Yet, the relatively rapid decline in statin adherence by 6 months suggests that it takes much less time to unlearn a habit. Future research should evaluate the use of "booster" interventions to achieve long-term benefits. In addition, longitudinal follow-up of adherence is needed in order to understand the long-term impacts of interventions. Novel methods, such as group-based trajectory modeling, can be used to model the dynamic nature of adherence over time. ${ }^{9}$ Furthermore, with an increasing medication burden on patients, providers will need to decide which medications to select for monitoring and how best to respond to the information collected. ${ }^{10}$ The use of an electronic monitoring bottle for a statin is one thing - but what about a patient on five medications of critical importance, some of which are weekly injections? In addition, it remains unclear which type of adherence feedback - individual or partner-is most impactful, since the two arms showed similar effects on adherence. Further research is needed to assess how best to integrate adherence information of all types (electronic, patient-reported, claims) into clinical practice and into patients' lives.

The authors conclude by asking "whether a continuous intervention that never ends could lead to sustained high levels of adherence." We would argue that high levels of adherence in the absence of improvements in important health outcomes should not be the ultimate goal. The current study showed that among a sample of patients with suboptimal adherence (MPR $<80 \%$ ), the overall mean baseline LDL was $88 \mathrm{mg} / \mathrm{dl}$. This suggests that it is possible to achieve low LDL levels with "suboptimal" adherence, and argues for the need to better define adherence thresholds that are able to discriminate across important health outcomes. Until then, the paper by Reddy and colleagues challenges us to "keep our eyes on the prize" of improving medication adherence and health outcomes.

Acknowledgments: Dr. Marcum is supported by funding from the Agency for Healthcare Research and Quality (AHRQ; K12HSO22982).

Corresponding Author: Walid F. Gellad, MD, MPH; VA Pittsburgh Healthcare System, University Drive (151C), Pittsburgh, PA, USA 15240 (e-mail: Walid.gellad@pitt.edu).

Compliance with Ethical Standards:

Conflict of Interest: Dr. Marcum has no relevant conflicts of interest to disclose.

Dr. Gellad serves as an unpaid member of the scientific advisory board for HealthPrize.

\section{REFERENCES}

1. Nieuwlaat $\mathbf{R}$, Wilczynski $\mathbf{N}$, Navarro $\mathbf{T}$, et al. Interventions for enhancing medication adherence. Cochrane Database Syst Rev 2014;CD000011.

2. Marcum ZA, Gellad WF. Medication adherence to multidrug regimens. Clin Geriatr Med. 2012;28:287-300.

3. Reddy A, Huseman TL, Canamucio A, et al. Patient and partner feedback reports to improve statin medication adherence: a randomized control trial. J Gen Intern Med. doi:10.1007/s11606-016-3858-0.

4. Long JA, Jahnle EC, Richardson DM, Loewenstein G, Volpp KG. Peer mentoring and financial incentives to improve glucose control in African American veterans. Ann Intern Med. 2012;156:416-24.

5. Conn VS, Ruppar TM, Enriquez M, Cooper P. Medication adherence interventions that target subjects with adherence problems: systematic review and meta-analysis. Res Social Adm Pharm. 2016;12:218-46.

6. El Alili M, Vrijens B, Demonceau J, Evers SM, Hiligsmann M. A scoping review of studies comparing the medication event monitoring system (MEMS) with alternative methods for measuring medication adherence. Br J Clin Pharmacol. 2016;82:268-79.

7. Bosworth HB, Fortmann SP, Kuntz J, et al. Recommendations for providers on person-centered approaches to assess and improve medication adherence. J Gen Intern Med 2016.

8. Stirratt MJ, Dunbar-Jacob J, Crane HM, et al. Self-report measures of medication adherence behavior: recommendations on optimal use. Transl Behav Med. 2015;5:470-482.

9. Lo-Ciganic WH, Donohue JM, Jones BL, et al. Trajectories of diabetes medication adherence and hospitalization risk: a retrospective cohort study in a large state Medicaid program. J Gen Intern Med. 2016;31:1052-60.

10. Steiner JF. Using adherence information to improve care: from clinic visits to populations. J Gen Intern Med 2016. 